\title{
Designing a Model of Interaction of Economic Resources in the Quantization Conditions of Economic Area
}

\author{
Elena Y. Shurkina \\ Kazan National Research Technical University named after A.N. Tupolev, Kazan, Russia \\ Email: frau.levina2010@yandex.ru \\ Elena V. Petrova \\ Galina D. Petrova \\ Chuvash State Pedagogical University named after I.Y. Yakovlev, Cheboksary, Russia \\ Lidiya V. Shirokova \\ Irina A. Astafyeva \\ Moscow State University of Mechanical Engineering, Moscow, Russia \\ Nadezhda Y. Gatzenbiller \\ Chuvash State Pedagogical University named after I.Y. Yakovlev, Cheboksary, Russia \\ Guzyal M. Kharisova
}

Kazan State University of Architecture and Engineering, Kazan, Russia

Alfiya R. Masalimova

Kazan (Volga region) Federal University, Kazan, Russia

\section{Doi:10.5901/mjss.2015.v6n2s3p129}

\section{Abstract}

The article aims to develop a methodology for investigating cyclic recurrence of economic phenomena in the quantization conditions of the modern economy. The article highlights the quantization levels and the adaptive possibilities of stabilizing the economic processes and phenomena. The authors have developed a target model of interaction of economic resources in the quantization conditions, allowing to identify the type and stages of interaction at the current time. The results of the research have a wide range of applications in the development of specific manifestations of economic regularities by researchers, practicians working in the field of economics.

Keywords: economic area, economic resources, quantization, modeling, interaction

\section{Introduction}

\subsection{Background}

The epicenter of the cyclical development is a crisis that carries the impetus for further development. The driving force of the economic cycle represents the impracticability of further development of economic systems at the existing level of production development within the boundaries of this scientific-technological paradigm. Consequently, the crisis, despite of all its negative consequences, carries out a positive role, introducing an element of self-development of the life process.

There is an obvious connection between technology and knowledge deficit and the natural resources aggravating 
scarcity at the level of the philosophy of unity and interaction features within the micro-, meso- and macro-levels, as factors of evolution. In our opinion, the main field of this interaction is the information market, which is integrated with the means of production, labor, government regulation and employment, becoming the main tool for achieving the advanced competitiveness. National priorities, arrayed in the spirit of knowledge-based economy, allow solving the facing pressing challenges, creating information-effective society, which at the same time becomes a conductor of new technological ideas, and a power to mobilize the hidden reserves of the economy.

\subsection{Status of the problem}

Development of scientific-technical and the related social process happens inconsistently, discretely for certain periods of socio-economic development caused by their own specific impulse, confirmed by statistical data on the growth rates of industrial production, changing sectoral structures, employment, and on the growth rates of GDP and national income, et cetera.

The specified problem had great significance for the development of the economy, which has led to its display in works of many economists of XIX and XX centuries. The activities of such scholars as MarX(1990), RodbertusYagetsov(2012), Mitchell (1927), Kitchin (1923), Schumpeter (1939) and others are devoted to research of economic cycles. In the works of these economists there are many different interpretations presented that determine the causes of cyclical development, as well as a variety of explanations of manifestations of the economic cycle and relatively adequate forecasts of economic activity (Dobrynin, Miropolsky, 1998; Simkina, 2000; Schumpeter, 1934).

Elements of the theory of quantum physics and their application to social and economic processes and phenomena are discussed by the authors Talbert, Bernstey (1995), Pan et all (2000), Blaug (2008), Shurkina (2014), Svirina et all (2014) and others.

\subsection{Hypothesis}

Actualization of an adequate system of integration into the information infrastructure, which should not only support the subjects of market relations in all areas of their work, but also provide an incentive for change and mobilization of domestic resources, stresses the need for the proper functioning of the market mechanism. Cyclicity in the form of economic dynamics is a continuous wave-like movement, alternating extensive and intensive types of development. Without a clear understanding of mechanisms underlying these changes it will be quite difficult to identify the manner in which the information infrastructure can and should be a source of resources for the modernization of the domestic industry, which is based on innovative processes. And, accordingly, to transfer to what is essentially new, the innovative attitude to information resources and technologies of their management should become one of the most important conditions for the accelerated development of the Russian economy, as we have seen it in the leading countries of the world.

\section{Materials and Methods}

\subsection{The definition of a quantum system}

In order to improve the processes of economic resources interaction in the socio-economic system, we will describe their combination in the form of a quantum system, i.e. in terms of states. Since in the foundation of the theory of quantum information underlies the principle of superposition of states, which says, that if a system can be in different states, then it can be in the states that are obtained by simultaneous "overlapping" of more than one state from this set, which fully reflects the activity of the socio-economic system.

We will represent a specific process in a form of a state vector, as it is a description of a closed system in the chosen basis. This description is given by the Hilbert space vector.

A wave vector, as a special case of the state vector, that is one of its coordinate representations - as the basis the spatiotemporal data are selected - can be used to directly specify the function of socio-economic process, not only with specifically selected parameters, but also with their particular values.

Any socio-economic process of economic resources interaction has inseparability (quantum entanglement), which implies the impossibility of separating the system into separate, autonomous and completely independent components.

Nonlocal correlations or quantum correlations are inseparability specific effect, consisting in a consistent behavior of individual parts of the system, which leads to the fact that modification of one part of the system simultaneously has an 
effect on all its other parts. In quantum physics the laws of quantum correlations are quantitatively described, which in turn makes it possible to obtain and analyze numerical evaluations of all without exception parameters (coefficients and immediate values) in the interaction within the system, describing the socio-economic processes in general.

Any socio-economic process is a system in which the principle of synergetics works, and, being directed to a particular object, represents a coherent state of individual processes, because it is a superposition of pure states, that is "overlapping each other" with different states of the existence of a closed system. Coherence in this case means the coordinated behavior of individual forming parts of the system by nonlocal correlations between them.

\subsection{State space formation of the economic system and processes}

Hilbert space, including a state space, that is, a set of all potential states of the system, represents all possible, developed, and as yet unknown variants of socio-economic processes with parameters for each individual case with the values of all possible parameters.

Since the set of all possible interacting with certain coefficients of options and their values is a system, then subsystems can be allocated, which represent specific processes with specific values.

Thus, the socio-economic process of economic resources interaction can be used with intricate in internal state concepts, decoherence of the overall structure of the economic resources interaction in connection with the violation of nonlocality (impossibility to compare local elements), as well as with a decrease in quantum entanglement between the forming parts of the economic system as a result of its interaction with the environment. Decoherence of the socioeconomic system is permissible due to its separability, that is, the possibility of separating the system parts as fully independent objects that can only be possible if there is lack of interaction between the system components.

Hilbert space of the processes of socio-economic systems is stored in the density matrix, using which it is possible to describe both the closed systems and the open ones, interacting with their environment. Any system in quantum theory corresponds to a certain state of the system, that is, the materialization of certain potentials of the system under these conditions. It is determined by a complex of measurable characteristics that manifest themselves, in particular, as a result of self-influence. The state of the system is defined by a state vector or density matrix. A part of the socio-economic processes are open systems, which are characterized by a mixed state, that is a state of the system, which cannot be described by a single state vector, but formalized only by the density matrix, that is the opposite of a pure state or a system isolation (it is a system state described by one state vector).

Since an entropy serves as a measure of disorder in a system, then the larger the number of admissible states a system has, the higher an entropy is. This is a major "minus" of the model.

\section{Results}

\subsection{Evaluating the measure of the economic resources' interaction}

Investigation of the interaction of economic resources at the level of a business agent means that its external and internal environment should be assessed in the conditions of uncertainty. Let us estimate the interaction of economic resources as a key internal factor in the development of an entrepreneurial agent as follows:

$$
E R_{i}=r_{i} \sum_{k=1}^{q} r_{i k} \psi_{i k}
$$

where $E R_{i}$ - is the number of i-th enterprise resource available at a given time, in cash or in kind; ${ }^{r}$ - is the number of $\mathrm{i}$-th resource, which an economic agent has in accordance with the deterministic approach; $\psi$ - the wave function that describes the state $r_{i}$, that is the characteristic of the resource used by the economic agent in each timepoint during the whole period of existence; $k(1 \div q)$ - is the resource number for a certain period.

The wave function implements the proposed by Heisenberg uncertainty principle for the processes of socioeconomic development, and allows to formalize a way for determining the level of uncertainty at each stage of the business cycle on the basis of an evaluation of internal and external environment of the enterprise.

For the purposes of this research, the data on the performance efficiency of nine companies (small and mediumsized businesses and corporations of different industry sectors: construction, processing and production of petroleum, chemical industry, services), which included a preliminary assessment on efficient use of resources, calculated as the 
ratio of profit to total resources consumption. The evaluation of the effectiveness of the resource interactions was carried out using deterministic and quantum models upon expiration of three months after the preliminary assessment using the financial data of the enterprises, as well as expert evaluations.

\subsection{Correlation analysis for deterministic, quantum and actual efficiency of interaction for the material and human resources}

The results of the Pearson correlation analysis for the material and human resources are presented respectively in Table 1 and Table 2.

Table 1. Pearson correlation for deterministic, quantum and actual efficiency of interaction between material resources

\begin{tabular}{|l|l|c|c|c|}
\hline Indicator & DEMR & QEMR & AEMR \\
\hline \multirow{3}{*}{ Efficiency material resources (deterministic model) DEMR } & Pearson correlation & 1 &, $919^{\star \star}$ &, $951^{\star \star}$ \\
\cline { 2 - 5 } & value & &, 000 &, 000 \\
\cline { 2 - 5 } & $\mathrm{N}$ & 9 & 9 & 9 \\
\hline \multirow{3}{*}{ Efficiency material resources (quantum model) QEMR } & Pearson correlation &, $919^{\star \star}$ & 1 &, $985^{\star \star}$ \\
\cline { 2 - 5 } & value &, 000 & &, 000 \\
\cline { 2 - 5 } & $\mathrm{N}$ & 9 & 9 & 9 \\
\hline \multirow{3}{*}{ Actual efficiency material resources AEMR } & Pearson correlation &, $951^{\star \star}$ &, $985^{\star \star}$ & 1 \\
\cline { 2 - 5 } & value &, 000 &, 000 & \\
\cline { 2 - 5 } & $\mathrm{N}$ & 9 & 9 & 9 \\
\hline$* \star$
\end{tabular}

The data in Table 1 show that the assessment of the efficiency of interaction of material resources provides a higher reliability of forecasting the future activities of the economic agent.

Table 2. Pearson correlation for deterministic, quantum and actual efficiency of interaction between human resources

\begin{tabular}{|l|l|c|c|c|}
\hline Indicator & DHMR & QHMR & AHMR \\
\hline \multirow{3}{*}{ Efficiency human resources (deterministic model) DHMR } & Pearson correlation & 1 &, $902^{\star *}$ &, $908^{* *}$ \\
\cline { 2 - 5 } & value & &, 000 &, 000 \\
\cline { 2 - 5 } & $\mathrm{N}$ & 9 & 9 & 9 \\
\hline \multirow{3}{*}{ Efficiency human resources (quantum model) QHMR } & Pearson correlation &, $902^{\star \star}$ & 1 & $1,000^{* \star}$ \\
\cline { 2 - 5 } & value &, 000 & &, 000 \\
\cline { 2 - 5 } & $\mathrm{N}$ & 9 & 9 & 9 \\
\hline \multirow{3}{*}{ Actual efficiency human resources AHMR } & Pearson correlation & $1,000^{* \star}$ &, $985^{* \star}$ & 1 \\
\cline { 2 - 5 } & value &, 000 &, 000 & \\
\cline { 2 - 5 } & $\mathrm{N}$ & 9 & 9 & 9 \\
\hline${ }^{* *}$ Correlation is significant at the 0.01 level & \multicolumn{3}{|c}{} \\
\hline
\end{tabular}

The data in Table 2 confirm the results of previous correlation analysis, therefore, the use of the quantum approach in assessing the interaction efficiency of economic resources makes it possible to obtain more accurate data in advance planning of the economic agent's activity.

\subsection{Quantitative assessment of information resources}

Effectivization of the economic entity, as a rule, leads to structural transformations in the management of resources of the economic entity. Modifications of the external environment actualize the need to assess anew the formed organizational communications of the management subjects and the operation of resources in the activities of the economic entity. Information resources that form a particular information field which enables the exchange of information between the economic entities, are becoming of paramount importance that, in the light of computer technology development, is leading to formation and development of the information technologies contributing to the management efficiency growth. Introduction of innovative information technologies intends to improve as a primary objective the collection, processing, storage, use, accumulation and submission of information, allowing to bring the information resource to a higher level of 
quality. In this context, dual nature is inherent in the information resource, which consists in the fact that it is, on the one hand, an independent resource on a par with the materials or personnel, and on the other hand - a management tool promoting the effectivization of management decision making.

Development of the information society has repeatedly increased the volumes of information circulating in the global economic space. Perception and use of the information requires the availability of a certain individual recipient's thesaurus $S_{g}$, reflecting the level of knowledge of the recipient, that is, a priori knowledge of the recipient. Clearly, this amount of information $I_{c}$. is nonlinearly dependent on the adequacy of the individual user thesaurus, and, despite the fact that the semantic content of the information message $S$ is constant, the recipients having different thesauri, will be getting unequal amounts of information. In case of proximity of the information recipient individual thesaurus in the considered sphere to zero $S_{1} \approx 0$, in this case, also the amount of information perceptions by the recipient will be equal to zero: $I_{c}=0$.

Possible is also the opposite marginal case, that is an individual recipient thesaurus $S_{r} \rightarrow \infty$, that is the recipient has absolute information about the analyzed subject, which leads to impossibility of further information generation. Respectively between the specified polar values of the thesaurus there is an optimal value, $S_{\text {ropt. }}$.

In the conditions of the economic space quantization it is proposed to estimate the quantity of information resource as a value that helps to achieve a set goal, relying on the statistical theory of Shannon and considering the quantity of information resource as an increment of the probability of achieving the goal. In case the probability of achieving the goal before the use of the information resource is equal to $P_{0}$, and after the use $-P_{1}$, then the quantity of information resource $I_{r}$ is defined as:

$$
I_{r}=\log \frac{P_{1}}{P_{r}} .
$$

The need for an information resource in the production of science-intensive and traditional products is increasing, and the inclusion of information in the price of the goods being traded involves also the consumer in the value chain, which is growing to the maximum of possible extent, and appearing in a variety of networks, hierarchically interconnected with various degrees of tightness and time of mutually beneficial operation. The views of different authors on the usefulness and value of the information resource are presented in the works by M. Koemtsi (Koemtsi et all, 2001). An information resource is only then good for the economic system, when there is usefulness in applying it to the process of current performance within the given system, that is, the utility level of the information resource determines its value. They claim that the Value of the information resource directly depends on the quality of the decisions made and the actions based on those decisions, that is, only the correctly used information resource is of value (Torrington, Wightman).

According to another author, the value of the information resource is defined as the maximum benefit that a certain amount of information can bring to the reduction in the average losses (Stratonovich, 1975).

Thus, we can assume that the value (Val) of the information resource represents a criterion of tangible and intangible effects acquired through the use of the information resource or as an increase in the degree of achieving the established goal:

$$
\text { Val }=\log _{2} \frac{P}{p} \text {, }
$$

where $p$ - is the probability of achieving the goal before the use of an information resource with a priori information; $P-$ is the probability of achieving the goal after the consumption of an information resource, in the case, if $P<p$. Consequently, Val - is a negative number, which tells about the transformation of information into misinformation. In the case of achieving the established goal, $\mathrm{P}=1$, the value of the information resource is maximum, therefore, $\mathrm{Val}=\mathrm{Val}($ max $)=\log 2 \mathrm{n}$.

An indispensable condition for achieving the goal is to use a modified formula:

$$
V a l=\frac{P-p}{1-p}
$$

where Val varies within [0-1]

In the case of equal probability of the events $p=1 / n$, where $n-$ is the number of variants, $p$ - normalized multiplier (Isaev, Nemchin, 2002).

The information resource value has a subjective character, as it is evolving, developing together with human society (the valuable becomes not valuable and vice versa), and depends largely on the recipient. The information resource consumption process is even more difficult for an assessment than its production, as the subjective nature of consumption is higher. The needs for an information resource are endless. However, if we assume that a consumer 
knows his information resource needs, the number of the needed information resource acquires foreseeable outlines. The need for an information resource increases, but at a decreased pace. This tendency is confirmed by the mathematical theory of information.

\section{Discussions}

The interaction of market participants are based on a continuous exchange of information, with that the distribution of the information flows across networks and the economic space requires a certain amount of time. Information is an essential component of any economic system. A system cannot exist without information support, since by virtue of the objective properties, information reduces the degree of its entropy, it structures the elements. Violations of information flows, lack of relevant information - lead to failure of systems, to decrease in their effective functioning. Economic actors, depending on the prevailing conditions, are constantly changing the operating parameters, which leads to inconsistencies in real state of objects, events, transaction terms and content of the requested, transmitted information. These reasons form the background distortion and asymmetry of the information field and causes subsequent inadequate, inefficient actions of market participants.

Some features of managing the economic systems are caused, in our opinion, by the quantization of the economic area, as well as by a manifestation of the quantum nature of economic processes at the level of economic systems in a state of instability, that is the economic systems open to external influences, changing under the influences in accordance with the response to the impulses representing an impact of the environment and directed to development of the system. Quantization of the economic space is characterized by the reaction of the system to a certain amount of the external impact impulse and allows to outline the level of inertia of the system, i.e. the duration of its existence in terms of external influences perception upon itself.

According to the author, the conducted research updates the application of the quantum theory elements, as well as the research methods and tools used in quantum physics, to determine the state of the elements of the economic space which possesses the quantum nature, and having restrictions on the measurability, as of the timepoint under analysis.

\section{Conclusions}

The need for an information resource in the production of science-intensive and traditional products is increasing, and the inclusion of information in the price of the goods being traded involves also the consumer in the value chain, which is growing to the maximum possible extent, and appearing in a variety of networks, hierarchically interconnected with various degrees of tightness and time of mutually beneficial operation.

Every extra unit of an information resource has a diminishing marginal utility, since the area of uncertainty with each new unit of information resource is reduced by half. Purchasing information under the marked assumptions is expedient as long as the marginal utility of each new piece of information is on par with the marginal costs for its acquisition. Thus, the complete elimination of uncertainty is inexpedient for economic agents, and they will be choosing the optimal level of uncertainty in accordance with the marginal utility of an extra unit of the information resource and the marginal costs for receiving it. The advantage of using the methods of quantum physics in consideration of functioning of the socio-economic systems is also in the ability to use simultaneously an unlimited number of parameters and a number of values for each, as well as in the instant productivity at manipulation with numerical values, which is associated with the use of capabilities of quantum information units - qubits or quantum bits, allowing to store simultaneously all of the above values.

The developing concepts on the basis of quantum physics allow the application of theoretical insights from the field of quantum information for modeling social and economic processes, that is due to the fact, that the quantum description is the most comprehensive of all the famous descriptions of reality, which will allow to increase reliability of advance planning for economic agents.

\section{Acknowledgments}

The authors thank all participants of this study for their kind cooperation. 


\section{References}

Blaug, M. (2008). Great Economists before Keynes: An introduction to the lives \& works of one handred great economists of the past. St. Petersburg Economicus publishing, 331.

Dobrynin, A.I., D. M. Miropolsky (1998). Balance and disbalance social and economic systems. St. Petersburg publishing house of Saint Petersburg State University of Economics and Finance, 67.

Isaev V.V., A.M. Nemchin (2002). The general theory of socio-economic systems. St. Petersburg Business Press publishing, 21.

Kitchin, J. (1923). Cycles and Trends in Economic Factors. Review of Economics and Statistics, 5 (1), 10-16.

Koemtsi, M., I. Teodorakioglu, J. Hadzhidimitriu (2001). Value and information management in modern enterprises. Enterprise Upgrading: factors and strategies (under. Ed. V.N.Eytingon). Voronezh State University publishing: 147-148.

Marx, K. (1990). Capital: Critique of Political Economy. London Penguin Books: 164-179.

Mitchell, W. (1927). Business Cycles: The problem and its setting. New York National Bureau of Economic Research publishing, $12-49$.

Pan, J.-W., D. Bouwmeester, M. Daniell, H. Weinfurter, A. Zeilinger (2000). Experimental test of quantum nonlocality in three-photon Greenberger-Horne-Zeilinger entanglement. Nature publishing, 403, 515.

Rodbertus-Yagetsov, J. K. (2012). Study on the capital: Trade crises and state economy. 2nd ed. Moscow LIBROKOM publishing: 9698.

Schumpeter, J. (1934). The Theory of Economic Development. - London Oxford University Press publishing, 13-45.

Schumpeter, J. (1939). Economic cycles: A theoretical, historical and statistical analysis of the Capitalist process. Eastford Martino Publishing, 43- 87.

Shurkina, E. (2014). The value of the information resource in the conditions of quantized economic space. Theory and practice of social development, 2, 382-384

Simkina, L.G. (2000). The human capital in innovative economy. Monography. St. Petersburg publishing house of St. Petersburg state engineering and economic academy, 11-16.

Stratonovich, R.L. (1975). Information Theory. Moscow Soviet Radio publishing, 424

Svirina, A., E. Parfenova, E.Shurkina, (2014). Evaluation of Uncertainty on the Stages of Business Cycle: Implementation of Quantum Principles. Journal on Systemics, Cybernetics and Informatics, V 12, 4, 79-85.

Talbert, J., Bernstey, B. (1995). Program reusable become reality. Making reuse a reality. Computerra Journal, 18, 13-15. 